\title{
Utility of adeno-associated viruses to target members of the TGF- $\beta$ superfamily in prostate cancer therapy
}

\author{
Priya Ramarao, Elspeth Gold \\ Department of Anatomy, University of Otago, Dunedin, New Zealand \\ Email: elspeth.gold@otago.ac.nz
}

Received 28 July 2013; revised 28 August 2013; accepted 15 September 2013

Copyright (c) 2013 Priya Ramarao, Elspeth Gold. This is an open access article distributed under the Creative Commons Attribution License, which permits unrestricted use, distribution, and reproduction in any medium, provided the original work is properly cited.

\begin{abstract}
Components of the TGF- $\beta$ superfamily have been well established in their intricate and multifaceted roles in cancer progression and survival. The TGF- $\beta$ s have been targeted therapeutically in an attempt to modify complex tumour networks to favour cancer cell destruction. Goals of these therapies are often to attack the "hallmarks" of cancer: characteristics acquired by cancer cells via re-wiring or manipulating existing biological pathways to their survival advantage. Of the multitude of targeted therapies currently available, viral therapies have shown much promise in their efficacy of treatment. This review highlights current viral therapies targeting members of the TGF- $\beta$ superfamily, with a focus on the strengths and limitations associated with this form of targeted cancer therapy.
\end{abstract}

Keywords: AAV; Prostate Cancer; TGF- $\beta$ Superfamily

\section{PROSTATE CANCER}

One of the leading causes of cancer related deaths in developed countries, prostate cancer, affects a large number of men. The progression of the cancer from its initiation in the prostate, which often precedes dispersion as metastases to tissues such as bone, lymph, liver and brain leaves several windows of opportunity during which certain therapies can be administered. While several treatment methods have been developed targeting a multitude of aspects of tumour characteristics, acquisition of resistance is a familiar roadblock encountered by investigators, and circumventing this obstacle remains a difficult task [1].

\section{ROLES OF THE TGF- $\beta$ S}

Despite partaking in a variety of developmental process- es crucial for normal development, aberrant reactivation of these TGF- $\beta$ s often results in tumorigenic behaviour [2]. The roles they partake in during tumourigenesis often reflect their roles in embryonic development, but also extends to other features often observed in cancer, such as cachexia and bone loss [3]. A re-deployment of their developmental roles to the advantage of the tumour is observed. At the stages of prostate tumourigenesis, increased TGF- $\beta$ production results in extracellular matrix degradation, immunosuppression and angiogenesis, all resulting in evasion of cell death and increased cell survival, favouring cancer cell propagation [4]. The conundrum faced when targeting TGF- $\beta$ is the fact that in the earlier stages of prostate tumourigenesis, TGF- $\beta$ exerts growth inhibitory effects on cells, which is a desirable characteristic in cancer treatment, whereas in later stages encourages tumour growth [4]. As such, understanding the underlying rewiring of pathways that occur before this fundamental switch are essential to capitalise favourable tumour suppressive roles of TGF- $\beta$, while simultaneously minimising its tumour promoting role.

\section{The TGF- $\beta$ Superfamily}

Members of the TGF- $\beta$ superfamily consist of TGF- $\beta$ s, BMPs, NODAL, activins and GDFs. NODAL influence the tumour parenchyma, whereas activins and GDFs have been found to modulate the tumour microenvironment.

The core of the TGF- $\beta$ signalling cascade is shared and similar across all TGF- $\beta$ superfamily members. Ligands of this superfamily dimerise either as homodimers or heterodimers, bridged by a disulphide bond. Receptors for these homo/hetero-dimers are a complex of two receptors, Type 1 and Type II, which also associate with each other to form a double complex. In its ligand bound state, the Type II receptor phosphorylates its partnered Type I receptor, thereby activating the kinase domain of the Type 1 receptor. As a result, downstream effectors such as 
SMADs are phosphorylated by the Type I receptor (SMAD2/3 for activins, TGF- $\beta$ s, GDFs and NODAL, SMAD1/5/8 for BMPs), and this conformational change allows the association with SMAD4/5. Nuclear import is made permissible by this association, and the complex is able to bind a variety of other proteins to fine-tune transcription (Figure 1).

SMADs recruit chromatin remodellers which are able to either activate or repress gene transcription, depending on whether they promote chromatin condensation or chromatin expansion. One of the many layers of regulation of SMAD activity include the upstream antagonists of the ligands. SMADs are phosphorylated at several different sites to modulate their activity; these modifications are catalysed by mediators of other signalling pathways such as the MAPK pathways [5-7]. NODAL is required for epithelial-to-mesenchymal transition as well as cell sorting. Intriguingly, the signalling strength is able to dictate the effect on surrounding cells. It has also been found that high levels of PI3K activity induce pluripotency, whereas low levels induce mesenchymal differentiation. These relate to Wnt and ERK signalling to pattern the anterio-posterior axis as well as the dorso-ventral axis. BMPs and GDFs are involved in maintaining and overseeing adult tissues, whereas NODAL is expressed embryonically; adult expression is often abnormal $[8,9]$.
The role of NODAL goes back to the very early stages of embryonic development, where it is involved in maintenance of pluripotency in the blastocyst. As a morphogen, it forms the basis of the proximal-distal axis, and in later stages, the anterior-posterior axis. Its role in pluripotency maintenance is also in conjunction with its ability to influence epithelial-to-mesenchymal transition: a key requirement for cells during development, to maintain the plasticity and alter the differentiation capabilities of the cells [10]. This plasticity, which comes hand in hand with increased proliferative abilities and regeneration is re-deployed during tumorigenesis. In fact, normal adult cells do not express NODAL; aberrant activation in adult cells are only observed in pathological conditions [11]. An example of this is in certain prostate cancers, elevated levels of NODAL are correlated with increased aggressiveness of the tumour, as well as increased potential for metastatic dissemination [12].

BMPs often oppose inducers of epithelial to mesenchymal transition. However, a re-wiring of BMP pathways see an overexpression of BMPs at the sites of metastasis. Intriguingly, in prostate cancer cells, BMP7 was able to maintain dormancy of a bone metastasis of prostate cancer. This effect was not applicable to the primary tumour, which shows that interaction with the tumour microenvironment may underpin this discrepancy [13].

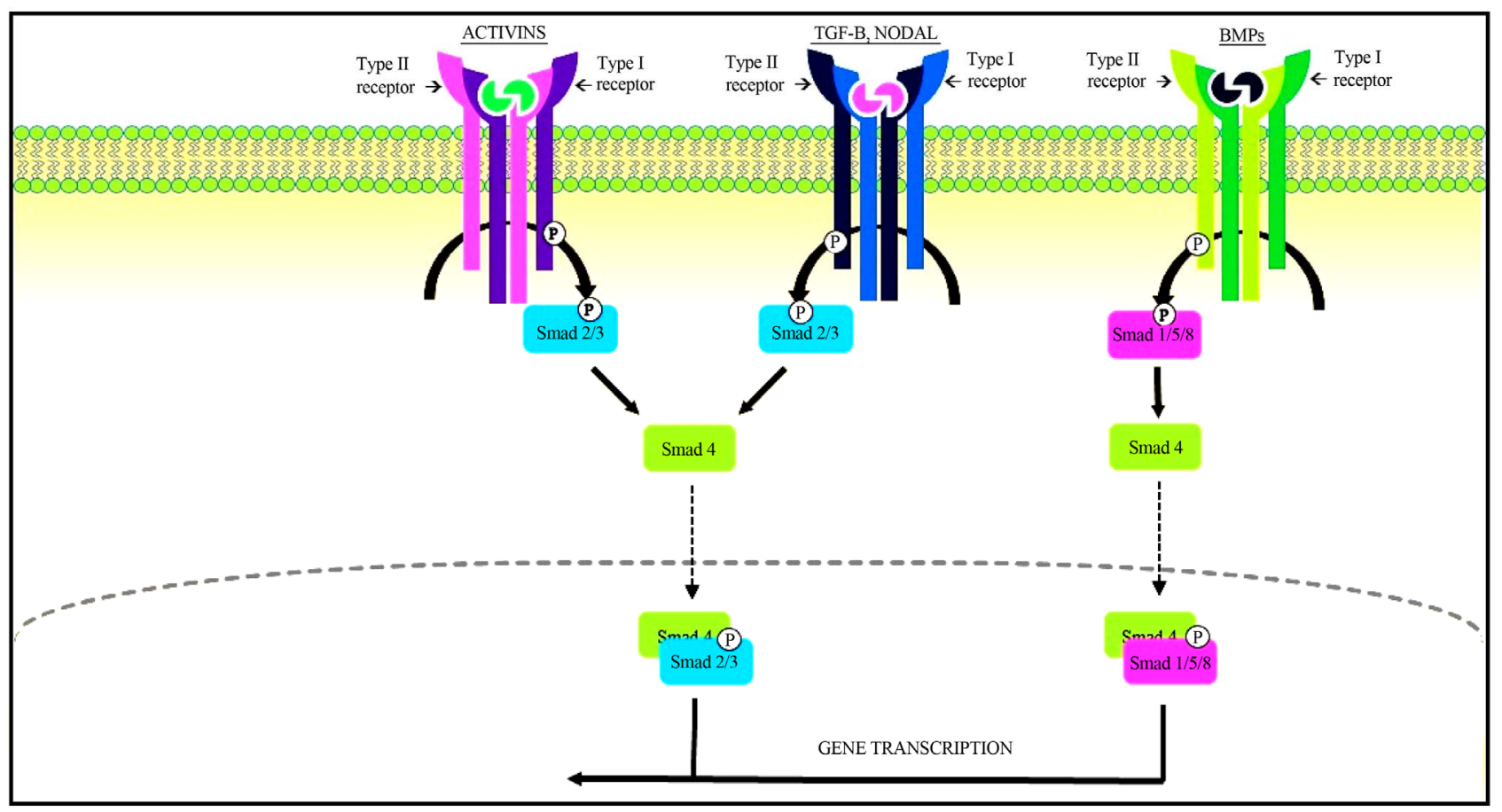

Figure 1. Signaling cascades of the TGF- $\beta$ superfamily_Each ligand binds their respective Type II receptor. Upon association of the ligand with its Type II receptor, phosphorylation of a Type I receptor is catalysed by the Type II receptor. Subsequently, the phosphorylated serine/threonine kinase domain of the Type II receptor in turn phosphorylates Smad 2 or Smad 3 (for activins, TGF- $\beta$ s, NODAL and GDF) or Smad1/5/8 for the BMP signaling cascade. Smad complexes which are formed are then able to bind Smad4, allowing for nuclear transport and transcription of relevant genes. 
Activins comprise two inhibin- $\beta$ subunits of varying subtypes; $\beta \mathrm{A}, \beta \mathrm{B}, \beta \mathrm{C}$ and $\beta \mathrm{E}$. The former two subunits are present in a diverse array of different tissues, whereas the latter two are found principally in the liver. Antagonists of these ligands are composed of an alpha subunit linked to a beta subunit via a single disulphide bond [14]. Redundancies exist between myostatin, activin and other TGF- $\beta$ superfamily members in terms of the consequence of their interactions with the activin receptors. Activins have also been discovered to be potent immunosuppressive agents, aiding tumours in immune evasion, another hallmark of cancer [15]. Increased activin levels, causative of a lowered inhibin antagonist level causes cancer cachexia, which is a wasting syndrome observed in many cancers. Activins and BMPs also play a role in balancing osleoclastic and osteoblastic activity, which are often aberrantly activated in instances of bone metastases [16].

Evidently, all the members of the TGF- $\beta$ superfamily are able to work synergistically to mold a favourable environment for tumorigenesis, by sculpting the tumour microenvironment. While their normal roles are crucial for development and regular homeostatic processes, aberrant reactivation or redeployment can result in highly unfavourable circumstances which promote tumour propagation and cancer progression. It is clear that the ability to target these components in a temporally controlled way can be of large benefit in cancer therapy to help restore the balance in the signalling pathways.

\section{POTENTIAL FOR THERAPEUTIC TARGETING OF TGF- $\beta$}

Dichotomous roles underpin TGF- $\beta$ signaling in relation to cancer progression; in early stages of tumorigenesis, TGF- $\beta$ signaling is endowed with the role of a tumour suppressor, whereas later stages see the signaling pathway involved in a more tumour promoting role. This multifaceted relationship poses as an obstacle to successful cancer therapies. Generally, therapies targeting the pathway can be directed at three points; at the ligand level, altering the dynamics of ligand-receptor interactions or modifying their second messengers.

\section{THE ROLE OF TGF- $\beta$ S IN PROSTATE CANCER}

The TGF- $\beta$ superfamily (made up of activins, TGF- $\beta$, BMPs, Nodal and GDFs) are involved in an intricate relationship in tumorigenesis, and teasing out the specific roles of this superfamily during cancer progression has yielded a wealth of information about their roles in cancer progression. In normal cells, TGF- $\beta$ s are growth inhibitors; they promote cell cycle arrest, a necessary step for growth control and apoptosis. It has been postulated that at this stage, signaling is carried out through a Smaddependent pathway, whereas metastatic dissemination is attributable to a non-Smad dependent pathway [7]. Additionally, there is a collaborative interaction between the androgen receptor and TGF- $\beta 1$, as the promoter region of TGF- $\beta 1$ contains androgen response elements, illustrating a synergistic control over growth processes. Insensitivity to the pro-apoptotic signals conferred by the TGF- $\beta$ signaling pathway is observed in instances whereby androgen receptor (AR) is overexpressed; even in hormone-independent prostate cancer, AR overexpression constitutes a dampened sensitivity to growth inhibitory signals elicited by TGF- $\beta$ pathways [17]. Induction of apoptosis is observed when TGF- $\beta 1$ is administered into the prostate, as well as an alteration from an epithelial to a mesenchymal nature (EMT), a hallmark of oncogenic transformation. Interestingly, the aforementioned transformation precedes dissemination of bone metastases [4].

Much research has been invested into deciphering the cues and components which facilitate the switching of TGF- $\beta$ from tumour suppressor to tumour promoter. Intriguingly, attenuation of the default TGF- $\beta$ pathway, with simultaneous reactivation and rewiring of TGF- $\beta$ controlled pathways is often observed in prostate cancer-manifesting as increased levels of TGF- $\beta$ ligands. As such, there is a clear fundamental connection between TGF- $\beta$ and different levels of cancer progression. This begs the question - what approaches can be used to target these components?

\section{TARGETING TGF- $\beta$ S WITH VIRUSES}

While antibody therapy [18] and antisense oligonucleotides [19] are commonly used therapies to target TGF- $\beta$ s, another method with superior targeting accuracy appears to be viral vectors [20]. The next section aims to discuss studies which have employed adenoviral-related approaches to target members of the TGF- $\beta$ superfamily, and the potential that these approaches may have when targeted to the prostate. Two main methods exist for viral targeting of cells; oncolytic viruses-which catalyse ubiquitous infection but rely on cancer cell-specific replication machinery, and are promoter driven. The former is achieved by selecting a promoter which is recognised by transcription factors and messengers only found in the cell type which is being targeted, and the latter by altering the tropism of the virus to target certain receptors found on the target cell type. In prostate cancer, PSA is an often-used promoter to drive viral integration or gene transcription. However, its dependency on androgens, owed to an AR-dependent enhancer poses as an obstacle when it is being used alongside androgen deprivation therapy [21]. 


\section{GENE THERAPY WITH VIRAL VECTORS}

\subsection{Adenoviruses}

Adenoviral vectors require target cells to express a CAR receptor for viral integration; CAR receptors are often overexpressed in prostate cancers. Despite this, different classes of prostate cancer show varying vulnerability to adenoviral infection. Addition of a cell-type specific molecule, such as an antibody or peptidecan deliver the viral content in a cell-targeted manner. A variety of specific motifs bind the CAR receptors; upon successful binding, the virus is assimilated into the cell, where it can carry out its oncolytic activity. While the main aim for viral delivery is to successfully infect target cells, it is equally vital to "detarget" the virus to ensure minimal delivery to normal, non-target cells [22]. As such, a wealth of research has been undertaken to pinpoint specific motifs which will catalyse infection in desired cells only. To this end, directed evolution of viruses is conducted to produce an array of serotypes able to target different tissues. The malleability of adenoviral vector fibre knobs make it customisable to be used for many tissues.

Two main methods exist for targeting viruses to preferred cells - transductional and transcriptional targeting. The former involves directing the virus to infect a limited set of cells, preferably cancer cells specifically. The latter results in ubiquitous infection, without differentiating between cancer cells and normal cells. Transcriptional targeting can be achieved in two ways. The first method utilises a virus with deleted genes required for viral replication, only to be compensated for in tumours cells. As such, the destructive effects are only "activated" in the tumour cells. Normal cells remain unharmed as they are unable to compensate for the missing genes. Secondly, the E1A gene, required for viral replication can be knocked out in the viral genome, but can be induced in the presence of a tumour cell-specific promoter. Adenoviral vectors can also be oncolytic: the mere act of adenoviral replication in target cells is sufficient to elicit "oncolysis"-cancer cell death [23].

\subsection{Targeting Bone Metastases in Prostate Cancer}

TGF- $\beta$ s are involved in osteoclastic differentiation and dissemination of bone metastases. Later stages of cancer progression result in these bone metastases, with few therapies available to successfully treat this phase of the disease. Hu et al. [24], developed oncolytic adenoviruses which simultaneously targeted TGF- $\beta$ via a protein. Downregulation of second messenger SMADs was observed upon infection of these cells. Authors postulated that while the targeted infectivity of the adenoviruses to cancer cells specifically was sufficient for oncolytic ac- tivity, antitumour activity was further enhanced by the effect of SMAD downregulation on the tumour microenvironment. Dispersion of signals initiated by overexpression of TGF- $\beta$ into the surrounding support environment was therefore successfully inhibited, dampening the growthpromoting effects of the microevironment. Consequently, the study solidified the long standing reputation of adenoviral vectors as a suitable source of tumour targeting. This is an intriguing form of "armed" oncolytic adenoviruses; "armed” because in addition to oncolytic capabilities of the virus, it harbours a further capacity to downregulate second messengers.

\subsection{Targeting Cancer Cells with RNA Interference}

Downregulation of components via RNA interference can be achieved by exploiting natural cellular responses to double stranded RNA (dsRNA) species-human cells always consider dsRNA to be foreign, as they are not normally present in the body. In a recent paper by Oh et al. [25], adenoviruses expressing $\mathrm{TGF} \beta-1$ short hairpin RNA(shRNA) were introduced into a metastatic prostate cancer cell line, DU-145, achieving a 98\% downregulation of TGF $\beta$ - 1 mRNA, with a concurrent decrease in corresponding protein levels. In contrast with the approach outlined in the previous section, which targeted the TGF$\beta$ signaling cascade at the receptor level, this approach targets TGF- $\beta$ at the ligand level. Intriguingly, this study showed that when TGF- $\beta 1$ was downregulated, a compensatory mechanism is elicited whereby TGF- $\beta 3$ is upregulated. Due to the fact that TGF- $\beta 1$ and TGF- $\beta 3$ have overlapping functions, this has profound implications for future therapies targeting the TGF- $\beta$ ligands, as compensatory mechanisms may hinder any anti-tumour therapies targeting TGF- $\beta$ ligands. Another very relevant finding in this study was the downregulation of both TGF- $\beta 1$ and TGF- $\beta 3$ alongside shRNA mediated degradation of TGF$\beta 2$ ligand. As such, in the interest of successful therapies targeting this family of ligands, TGF- $\beta 2$ may be a more successful target to ensure faithful downregulation of all ligands that may have the same function.

\subsection{Utilising Immunological Effects to Elicit Anti-Tumour Responses}

A few studies have attempted to take advantage of the immunological response elicited by adenoviruses to provoke cancer cell destruction. The induction of active immunity is highlighted in two prominent studies. Firstly, inducing oncolysis by means of a suicide gene, Ad5-CD/ TK-rep conjures an immunological response which targets cells infected by the virus, thus selectively destroying cancer cells [26]. Another promising study [27] provokes T-cells to set up immunological responses to an 
adenovirus containing the PSA promoter, in hopes that the reaction set up by this process will destroy all PSA expressing cells. In terms of what this means for TGF- $\beta$ targeting, the above strategies can be conducted alongside a TGF- $\beta$ knockdown (at either the receptor, ligand or second messenger level) to produce a powerful antitumour response. Furthermore, background active immunity may also offer protection from potential future development of prostate cancer.

\subsection{Specific Targeting to Newcastle Disease Virus}

Another fascinating discovery was the use of a Newcastle Disease Virus (NDV) to specifically target prostate cancer cells. Incorporation of the virus was dependent on presence of a substrate (HSSKLQ), which is PSA specific. Its oncolytic activity was observed even in hormone refractory prostate cancer [28].

\section{ADENO-ASSOCIATED VIRUSES}

The very ability of adenoviruses to induce powerful immunological responses may also serve to be their downfall; with these responses also potentially preventing survival of the adenovirus for a duration long enough to carry out infection and subsequent tumour destruction. While adenoviral virus-based treatments have dominated the field of gene therapy, Adeno-Associated Virus (AAVs) are a much more attractive means of achieving tumour regression, notwithstanding a few drawbacks. They belong to a class of dependovirus, requiring the replication capability of a "helper" virus, such as adenovirus. In the absence of a helper virus, they succumb to a lysogenic cycle; they predictably incorporate at a locus on chromosome 19 (19q13.3), and then replicate alongside the host cell genome. When a helper virus is present, the AAV enters a lytic cycle, resulting in rapid replication and dispersion of progeny to surrounding cells.

Utility of AAVs in the clinic come with significant advantages. Weak immune responses are elicited by AAVs, making it an attractive candidate for gene therapy. Powerful immune responses such as those that can be elicited by adenoviruses can be counterproductive as well as dangerous to the host. In addition, inflammatory responses mediated by AAVs are far weaker than those induced by adenoviruses. The predictable nature of its integration locus avoids any concern for random insertion and mutagenesis. Despite these advantages, certain features of AAVs pose as obstacles to successful clinical utility. AAVs have a much lower infection efficiency compared to adenoviruses; $40 \%$ vs almost $100 \%$. Its packaging capacity is also half of that of adenoviruses. Also, because AAVs only package single stranded DNA, second strand synthesis is required after infection, although this has now been circumvented by self-complementary Adeno-Associated Viruses (scAAVs). Lastly, much like adenoviruses, peptides embedded in the viral coat of AAVs can be altered to direct them into a wide variety of tissue types, such as prostate. The variety of prostate-specific genes and antigens make it an ideal tissue for specific viral targeting, although there is often no differentiation between prostate cancer cells and normal prostate cells. However, as prostate cancer most often affects the post-reproductive population, this is not a huge obstacle. The following section aims to highlight some studies which have targeted TGF- $\beta$ s with AAVs, and subsequently evaluate the advantages of AAV-based TGF- $\beta$ targeting in prostate cancer.

\subsection{AAVs for siRNA Mediated Silencing of Androgen Receptor (AR)}

Although no studies have thus far attempted to target TGF- $\beta$ s with AAVs in prostate cancer, several other genes, such as the androgen receptor, have been targeted for downregulation in attempt to catalyse tumour destruction. The effectiveness of this procedure was exhibited in a study by Sun et al. [29], where short interfering RNA (siRNA) was packaged in an AAV and injected intratumourally as well as systemically. Delivery of siRNA targeting AR successfully downregulated the growth of prostate cancer xenografts. This has great potential for targeting the members of the TGF- $\beta$ superfamily - the fact that reliable infection of the virus into the prostate shows exciting potential for packaging a TGF- $\beta$ superfamily member to the virus to target the TGF- $\beta$ signaling cascades.

\subsection{ProstAtak}

An intriguing form of adenoviral associated therapy that utilises the suicide gene method is ProstAtak. Suicide gene therapy entails specific delivery of drug metabolizing enzymes into tumour cells. In the presence of a ubiquitously delivered prodrug, precise metabolism of these prodrugs into harmful metabolites is provoked in the cancer cells, thus selectively killing them. ProstAtak exploits targeted delivery of the herpes simplex virus thymidine kinase (HSV-tk) into cancer cells, in conjunction with the prodrug valacyclovir. This vaccine is currently under Phase III human clinical trials, with very promising preliminary results. The synergistic effects of ProstAtak and radiation therapy incite a stimulatory effect on the immune system, which poises it to attack remaining residual cancer cells. Immunotherapies such as this are becoming increasingly popular, as intrinsic "radar" systems set up by the body itself and may be a reliable and quick detection system which will lead to subsequent destruction of these "runaway" cells, regardless of where in 
the body they might be. Interestingly, AAV-mediated HSV-tk also shows potential in therapies which use gancyclovir in bladder cancer [30].

\subsection{GVAX}

Another promising clinical trial uses granulocyte macrophage colony-stimulating factor (GM-CSF) to promote cancer cell death. GM-CSF incites a potent immunostimulatory response which draws the attention of the immune system to the cancer cells. This factor has been used in a variety of clinical trials for different cancers, including bladder and prostate cancers. However, GVAX is a whole cell vaccine, which has been transduced by a recombinant AAV (rAAV) vector. Although this is not a viral vaccine, it does highlight the versatility of using AAVs to develop various therapies using their role as a gene transfer vector. GVAX is currently transitioning from Phase I to Phase II human clinical trials, with very encouraging results published in February 2012. A fixed dose of GVAX was conducted in conjunction with a dose escalation trial of Ipilimumab, a CTLA4-blocking antibody, for patients with metastatic castration-resistant prostate cancer (mCRPC) [31].

\section{CONCLUDING REMARKS}

It is clear that AAVs pose significant advantages and potential in cancer gene therapy. Although TGF- $\beta$ s have not yet been targeted with AAVs in prostate cancer, there is compelling evidence that this may be a promising therapy for metastatic, end stage prostate cancer. Overall, the high safety profile and ability to produce long term gene expression make AAVs a great potential therapy for mCRPC. At present, several AAVs have been rigorously interrogated for use in clinical applications; AAV8 for Haemophilia and macular degeneration, AAV1, 6 and 9 for cardiomyopathies, AAV5 for cystic fibrosis and AAV1, 5 and 8 for CNS-related diseases such as Parkinson's and Alzheimer's disease.

Despite the vast array of advantages involved with the utility of AAVs for cancer therapy, the transition of these therapies from the bench to the clinic is far from smooth. For example, although pre-clinical results showed encouraging potential for the use of AAVs in Haemophilia $B$, it was difficult to achieve therapeutic levels of Factor IX. Additionally, there was actually a T-cell mediated response elicited which resulted in the destruction of the therapeutic transduced cells [32]. This shows that although the immune responses elicited by AAVs are weaker in comparison to other viruses, it is sufficient to hinder appropriate delivery of the therapy. A valuable point to note is the fact that this immune response was not observed in mouse models [33], further highlighting the difficulties of translating therapies into the clinic.

\section{REFERENCES}

[1] McEntee, M.F., Ziegler, C., Reel, D., et al. (2008) Dietary $n-3$ polyunsaturated fatty acids enhance hormone ablation therapy in androgen-independent prostate cancer. American Journal of Pathology, 173, 256-268. http://dx.doi.org/10.2353/ajpath.2008.070989

[2] Chu, G.C., Dunn, N.R., Anderson, D.C., et al. (2004) Differential requirements for Smad4 in TGFB-dependent patterning of the early mouse embryo. Development, 131, 3501-3512. http://dx.doi.org/10.1242/dev.01248

[3] Fields, S.Z., et al. (2013) Activin receptor antagonists for cancer-related anaemia and bone disease. Expert Opinion on Investigational Drugs, 22, 87-101. http://dx.doi.org/10.1517/13543784.2013.738666

[4] Levy, L. and Hill, C.S. (2005) Smad4 dependency defines two classes of transforming growth factor B (TGF-B) target genes and distinguishes TGF-B induced epithelialmesenchymal transition from its antiproliferative and migratory responses. Molecular and Cellular Biology, 25, 8108-8125.

http://dx.doi.org/10.1128/MCB.25.18.8108-8125.2005

[5] Attisano, L. and Wrana, J.L. (2002) Signal Transduction by the TGF-B superfamily. Science, 296, 1646-1647. http://dx.doi.org/10.1126/science.1071809

[6] Heldin, C.H., Miyazono, K. and Dijke, P.T. (1997) TGFB signalling from cell membrane to nucleus through SMAD proteins. Nature, 390, 465-471.

http://dx.doi.org/10.1038/37284

[7] Derynck, R. and Zheng, Y.E. (2003) Smad-dependent and Smad-independent pathways in TGFB family signalling. Nature, 425, 577-584. http://dx.doi.org/10.1038/nature02006

[8] Ramel, M.C. and Hill, C.S. (2012) Spatial regulation of BMP activity. FEBS Letters, 586, 1929-1941. http://dx.doi.org/10.1016/j.febslet.2012.02.035

[9] Mesnard, D., Guzman-Ayala, M. and Constam, D.B. (2006) Nodal specifies embryonic visceral endoderm and sustains pluripotent cells in the epiblast before overt axial patterning. Development, 133, 2497-2505.

[10] Ogawa, K., et al. (2007) Activin-Nodal signalling is involved in propagation of mouse embryonic stem cells. Journal of Cell Science, 120, 55-65. http://dx.doi.org/10.1242/jcs.03296

[11] Wu, Z., et al. (2008) Combinatorial signals of activin/nodal and bone morphogenic protein regulate the early lineage segregation of human embryonic stem cells. The Journal of Biological Chemistry, 283, 24991-25002. http://dx.doi.org/10.1074/jbc.M803893200

[12] Topczewska, J.M., et al. (2006) Embryonic and tumorigenic pathways converge via nodal signalling: Role in melanoma aggressiveness. Nature Medicine, 12, 925-932. http://dx.doi.org/10.1038/nm1448

[13] Bujis, J.T., et al. (2007) BMP7, a putative regulator of epithelial homeostasis in the human prostate, is a potent inhibitor of prostate cancer bone metastasis in Vivo. Ame- 
rican Journal of Pathology, 171, 1047-1057. http://dx.doi.org/10.2353/ajpath.2007.070168

[14] Kingsley, D.M. (1994) The TGF-beta superfamily: New members, new receptors, and new genetic tests of function in different organisms. Genes \& Development, 8, 133-146. http://dx.doi.org/10.1101/gad.8.2.133

[15] Massague, J. and Gomis, R.R. (2006) The logic of TGFB signalling. FEBS Letters, 12, 2811-2820. http://dx.doi.org/10.1016/j.febslet.2006.04.033

[16] Katusno, Y., et al. (2008) Bone morphogenetic protein signalling enhances invasion and bone metastasis of breast cancer cells through Smad pathway. Oncogene, 27, 63226333. http://dx.doi.org/10.1038/onc.2008.232

[17] Kang, H.Y., et al. (2001) From transforming growth factor-B signalling to androgen action: Identification of Smad3 as an androgen receptor coregulator in prostate cancer cells. PNAS, 90, 3018-3023. http://dx.doi.org/10.1073/pnas.061305498

[18] Iyer, S., et al. (2005) Targeting TGF-B Signaling for Cancer Therapy. Cancer Biology \& Therapy, 4, 261-266. http://dx.doi.org/10.4161/cbt.4.3.1566

[19] Schlingensiepen, K.H., et al. (2004) The TGFbeta1 antisense oligonucleotide AP 11014 for treatment of nonsmall cell lung colorectal and prostate cancers: Preclinical studies. Journal of Clinical Oncology, 22, 31-32.

[20] Seth, P., et al. (2006) Development of oncolytic adenovirus armed with a fusion of soluble transforming growth factor B receptor II and human immunoglobulin Fc for breast cancer therapy. Human Gene Therapy, 17, 11521160. http://dx.doi.org/10.1089/hum.2006.17.1152

[21] Thalmann, G.N., et al. (2000) LNCaP progression model of human prostate cancer: Androgen-independence and osseous metastasis. Prostate, 44, 91-103. http://dx.doi.org/10.1002/1097-0045(20000701)44:2<91:: AID-PROS1>3.0.CO;2-L

[22] Lasaro, M.O. and Ertl, H.C. (2009) New insights on adenovirus as vaccine vectors. Molecular Therapy, 17, 13331339. http://dx.doi.org/10.1038/mt.2009.130

[23] Khare, R., et al. (2011) Advances and future challenges in adenoviral vector pharmacology and targeting. Current Gene Therapy, 11, 241-258. http://dx.doi.org/10.2174/156652311796150363

[24] Hu, Z., et al. (2012) Systemic delivery of oncolytic adenoviruses targeting transforming growth factor-b inhibits established bone metastasis in a prostate cancer mouse model. Human Gene Therapy, 23, 1-12. http://dx.doi.org/10.1089/hum.2012.040

[25] Oh, S., et al. (2013) Transforming growth factor-B gene silencing using adenovirus expressing using adenovirus expressing TGF-B1 or TGF-B2 shRNA. Cancer Gene Therapy, 20, 94-100. http://dx.doi.org/10.1038/cgt.2012.90

[26] Freytag, S.O., et al. (2003) Phase I study of replicationcompetent adenovirus-mediated double-suicide gene therapy in combination with conventional-dose three-dimensional conformal radiation therapy for the treatment of newly diagnosed, intermediate-to high-risk prostate cancer. Cancer Research, 63, 7497-7506.

[27] Lubaroff, D.M., et al. (2006) Clinical protocol: Phase I study of an adenovirus/prostate-specific antigen vaccine in men with metastatic prostate cancer. Human Gene Therapy, 17, 220-229.

[28] Shobana, R., et al. (2013) Prostate-specific antigen-retargeted recombinant newcastle disease virus for prostate cancer virotherapy. Journal of Virology, 87, 3792-3800. http://dx.doi.org/10.1038/cgt.2012.90

[29] Sun, A., et al. (2009) Adeno-associated virus-delivered short hairpin-structured RNA for androgen receptor gene silencing induces tumour eradication of prostate cancer xenografts in nude mice: A preclinical study. Cancer Therapy, 126, 764-774.

[30] Pan, J.G., et al. (2012) The adeno-associated virus-mediated HSV-TK/GCV suicide system: A potential strategy for the treatment of bladder carcinoma. Medical Oncology, 29, 1938-1947. http://dx.doi.org/10.1007/s12032-011-0091-x

[31] Eertwegh, A.J.M., et al. (2012) Combined immunotherapy with granulocyte-macrophage colony-stimulating factor-transduced allogeneic prostate cancer cells and ipilimumab in patients with metastatic castration-resistant prostate cancer: A phase 1 dose-escalation trial. Lancet Oncology, 13, 509-517. http://dx.doi.org/10.1016/S1470-2045(12)70007-4

[32] Manno, C.S., et al. (2003) AAV-mediated factor IX gene transfer in skeletal muscle in patients with severe haemophilia B. Blood, 101, 2963-2972. http://dx.doi.org/10.1182/blood-2002-10-3296

[33] Manno, C.S., et al. (2006) Successful transduction of liver in haemophilia by AAV-Factor IX and limitations imposed by the host immune response. Nature Medicine, 12, 342-347. http://dx.doi.org/10.1038/nm1358 